Mesenteric lipodystrophy

\author{
M S Bashir, C R Abbott
}

\begin{abstract}
Three cases of mesenteric lipodystrophy with a wide range of clinicopathological features are reported. Mesenteric lipodystrophy may present as an acute abdomen or with non-specific upper abdominal symptoms. Routine biochemical and haematological investigations are within normal limits. Histological examination shows lipid-filled macrophages in sheets and bands with focal cyst formation.

Mesenteric lipodystrophy is a rare condition. A firm diagnosis can be reached only by histological examination and a number of conditions need to be considered in the differential diagnosis.
\end{abstract}

(F Clin Pathol 1993;46:872-874)

Mesenteric lipodystrophy is non-specific inflammation of the adipose tissue of the mesentery. It was first cited in the English language by Durst et $a l^{1}$ and initially named retractile mesenteritis. In 1955 Crane et al ${ }^{2}$ described five cases showing the histological features of mesenteric lipodystrophy and called it isolated lipodystrophy. The term mesenteric panniculitis was also used to describe the histological features of this condition by Ogden, Bradburn, and Rives. ${ }^{3}$ Mesenteric lipodystrophy has been called early Whipple's disease, mesenteric variant of Weber-Christian disease, liposclerotic mesenteritis and mesenteric lipogranuloma. ${ }^{4}$ The histological features of all these cases are uniform and the term mesenteric lipodystrophy is favoured, as it describes a degeneration of fat and avoids the suggestion that the disease is a primary inflammation of adipose tissue.

Clinically, mesenteric lipodystrophy is a disease of late adulthood with a median age at presentation of 60 years ${ }^{4}$ and an age range of 20-80 years. It may be entirely asymptomatic, being discovered as a mass in the abdomen during examination or surgery for some unrelated condition. In patients with symptoms the commonest complaint is abdominal discomfort usually in the upper abdomen and varying in severity from a dull ache to sharp excruciating pain. Other symptoms usually associated with mesenteric lipodystrophy are anorexia, nausea, vomiting, diarrhoea, constipation and weight loss. ${ }^{4}$ Presentation as an acute abdomen has also been reported. ${ }^{5}$ The duration of symptoms may vary from two weeks to several years. ${ }^{1}$ Mesenteric lipodystrophy has also been described in the colon ${ }^{6}$ where it seems to be clinicopathologically more advanced and progressive. These patients usually present with abdominal pain, diarrhoea, constipation and a lower abdominal mass. Barium enema may show narrowing and poor extensibility of the colon. Computed tomograms and ultrasonography may show thickening of the mesocolon and colonic wall. The gross and microscopic features in the colon are similar to those seen in the small bowel mesentery.

The commonest physical finding is that of a deep-seated, poorly defined intra-abdominal mass. In those presenting with an acute abdomen, abdominal distension and tenderness are additional findings. ${ }^{56}$ The results of haematological and biochemical investigations are usually normal or non-specific and unhelpful in diagnosis. A computed tomogram or ultrasonography, however, may show a discrete mass or mesenteric thickening. In acute presentation a routine $x$-ray picture of the abdomen may show small bowel obstruction. The only definite way to diagnose mesenteric lipodystrophy is through biopsy.

The gross appearance at surgery may be of diffuse mesenteric thickening, a single discrete tumour, or multiple discrete tumours. In cases of diffuse mesenteric thickening the cut surface has a dirty grey to yellow fatty colour and may extend into the retroperitoneum. Discrete tumours may vary from $1-10 \mathrm{~cm}$ in size with a firm to rubbery consistency and a pale yellow to orange fleshy cut surface. In more advanced cases the cut surface may show multiple cysts filled with a dirty oily fluid. ${ }^{4}$

Histologically, the mesentery is infiltrated by lipid filled macrophages which may be confined by narrow septa within the adipose tissue or form broad sheets infiltrating the mesentery. Usually there is a sparse lymphocytic infiltrate but no evidence of acute inflammation or vasculitis. In more severe cases there may be areas of cystic necrosis surrounded by lipid-filled macrophages with an occasional multinucleated giant cell and longstanding cases may show some areas of fibrosis. The presence of necrosis and fibrosis increases proportionately to the degree of infiltration by lipid filled macrophages.

We present three cases showing the clinical and pathological spectrum of features. 


\section{Case reports}

CASE 1

A 65 year old man presented urgently with a two day history of nausea and vomiting. On examination he had a distended tender abdomen with absent bowel sounds. On radiological examination of the abdomen, multiple fluid and gas levels were seen. All the routine laboratory investigations were within normal limits. The patient underwent a laparotomy for acute intestinal obstruction which disclosed a $5 \mathrm{~cm}$ diameter mass in the mesoappendix with an adherent loop of small bowel. No other intra-abdominal pathology was found, the mass was removed, and the abdomen closed. This postoperative recovery was unremarkable.

Grossly, the oval, well circumscribed mass was firm in consistency, had a yellow orange cut surface, and contained multiple cysts up to $1 \mathrm{~cm}$ in diameter filled with dirty milky fluid. Microscopic examination showed infiltration of mesenteric fat with sheets and bands of lipid filled macrophages. The cysts associated with fat necrosis were filled with fat debris and lined by foamy macrophages, with an occasional multinucleated giant cell (figure). Bands of collagen were also present along with a small increase in the number of lymphocytes.

\section{CASE 2}

A 67 year old woman presented with a 15 year history of diarrhoea. Extensive investigations failed to give any clue as to aetiology. A jejunal biopsy specimen suggested the possibility of a lymphoma, but laparotomy and splenectomy failed to provide histological evidence of this. She was treated symptomatically. She died of pulmonary embolism from thrombosis of calf veins. At necropsy there was no abnormality in the gastrointestinal tract and no evidence of lymphadenopathy or

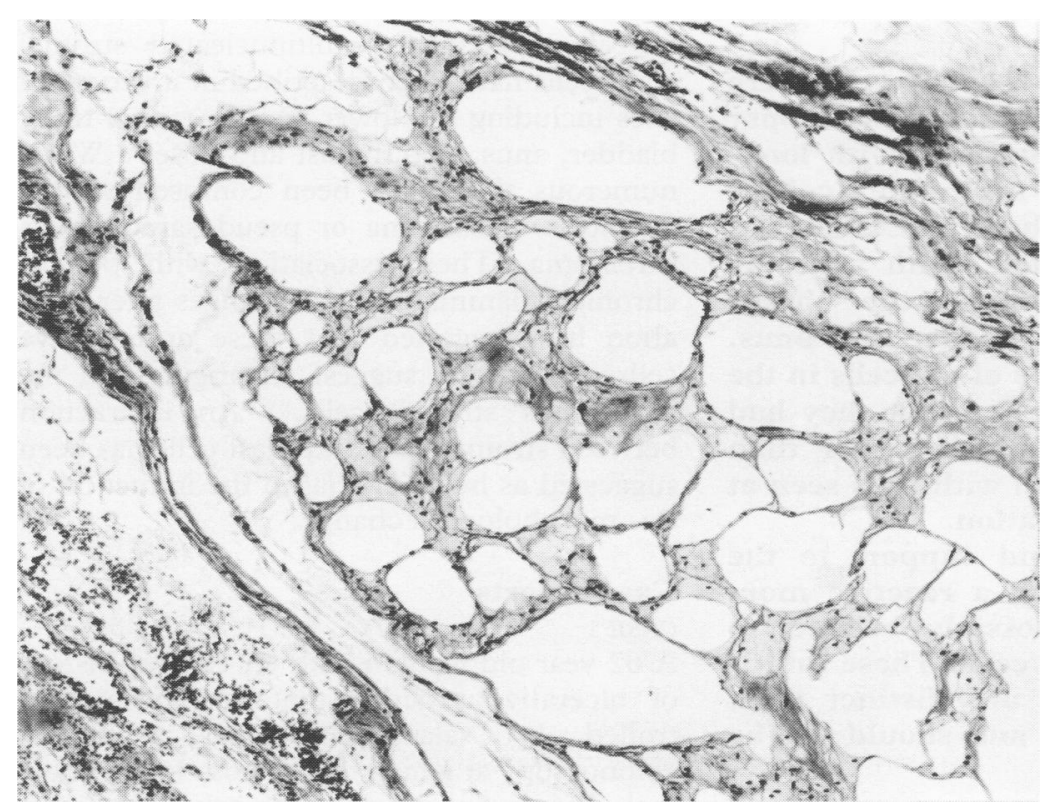

Infiltration of mesenteric fat with lipid-filled macrophages (haematoxylin and eosin). lymphoma was seen. Multiple nodules, measuring up to $3 \times 3 \mathrm{~cm}$ in the small bowel mesentery were noted, however, and on section these had a yellow surface with cystic areas filled with yellowish liquid debris. Histologically, the cystic areas were related to fat necrosis and the surrounding mesenteric fat contained large numbers of lipid filled macrophages. There was a mild lymphocytic reaction but no evidence of acute inflammation, vasculitis, or neoplasia.

\section{CASE 3}

A 55 year old man had a two month history of nausea, upper abdominal pain, and weight loss. He was thoroughly investigated for his symptoms. All investigations including a Barium meal follow through were within normal limits. Subsequently he developed bronchopneumonia and died. At necropsy apart from bronchopneumonic consolidation of the lungs, the only other finding was that of multiple nodules in the mesentery measuring 0.3 to $1.5 \mathrm{~cm}$ in diameter.

The nodules on section contained cystic areas containing brownish yellow oily liquid. Histologically, the nodules comprised cysts filled with debris. The surrounding mesenteric fat was diffusely infiltrated with sheets and bands of foamy macrophages with focal collection of lymphocytes. There was no evidence of any vascular or acute inflammatory process.

\section{Discussion}

The three cases of mesenteric lipodystrophy presented here demonstrate the clinical and pathological features of this condition. The clinical features are non-specific and routine investigations are unhelpful in diagnosis. The diagnosis of mesenteric lipodystrophy requires biopsy of the mesenteric lesions and several conditions need to be considered in the differential diagnosis.

Grossly, the mesentric swellings may simulate a lipoma, liposarcoma, or benign fibrous xanthoma. Benign fibrous tumours may be seen in the mesentery after abdominal surgery in Gardner's syndrome or familial polyposis ${ }^{7}$ and may be confused with mesenteric lipodystrophy. Intra-abdominal carcinoma, inflammation, or visceral perforation may be associated with acute fat necrosis and inflammation without a predominant lipid-filled macrophage infiltration.

A foreign body reaction after surgery or trauma may cause a mass with giant cells and foamy macrophages. Ingestion of paraffin oils from diet or laxatives may present as a nodular mass, ${ }^{8}$ but the nodule is made up of fibrous, frequently calcified cysts, filled with clear oil; there are usually extensive fibrous adhesions and although foreign body giant cells and fibrosis are prominent, there is relatively little infiltration by lipid laden macrophages. Weber-Christian disease may also mimic mesenteric lipodystrophy ${ }^{4}$ but the clinical features and a predominantly lymphocytic infiltrate aid in the differential diagnosis. 
Retractile mesenteritis, characterised by shortening of the mesentery with fibrosis and lymphocyte infiltration, has been suggested as a condition similar to mesenteric lipodystrophy, and some authors would regard it as an end stage of mesenteric lipodystrophy. ${ }^{9}$ An association between mesenteric lipodystrophy and retroperitoneal fibrosis has also been suggested and obstruction of the vena cava due to retroperitoneal extension of mesenteric lipodystrophy has been reported. ${ }^{410}$

In pelvic lipomatosis there is a gross increase in perivesical and perirectal adipose tissue but there is no foamy macrophage infiltration and the process does not extend above the pelvic brim. Although there may be inflammatory changes in the serosa in Whipple's disease, the mesenteric nodules represent enlarged lymph nodes which contain numerous periodic acid Schiff positive macrophages and bacilli and there is no sign of fat necrosis.

The three cases that we have presented demonstrate the clinical spectrum of mesenteric lipodystrophy. Case 1 presented with an acute abdomen and in cases 2 and 3, despite extensive investigations, the diagnosis was made at necropsy. Only histological examination of the mesenteric masses indicated the diagnosis.
The clinical course of mesenteric lipodystrophy is usually benign with a favourable outcome; no treatment is necessary. ${ }^{4}$ In the mesocolon the disease is clinicopathologically more advanced and progressive and may require surgical treatment, including a colectomy. ${ }^{6}$

It is important for pathologists and their clinical colleagues to be aware of mesenteric lipodystrophy as a cause of a mass in the abdomen when symptoms of anorexia, nausea, vomiting, diarrhoea and constipation are present.

1 Durst $\mathrm{AL}$ Freund $\mathrm{H}$, Rosenmann E, Birnbaum D. Mesenteric panniculitis: Review of the literature and presentation of cases. Surgery 1977;81:203-11.

2 Crane JT, Aguilare MJ, Grimes OF. Isolated lipodystrophy, a form of mesenteric tumour. Am F Surg 1955; 90:169-79.

3 Ogden WW, Bradburn DM, Rives JD. Mesenteric panniculitis. Ann Surg 1965;161:864-73.

4 Kipfer RE, Moertel CG, Dahlim D. Mesenteric lipodystrophy. Ann Intern Med 1974;80:582-8.

5 Shah AN, You CH. Mesenteric lipodystrophy presenting as an acute abdomen. Southern Med f 1982;75:1025-26. as an $Y$, Mori $M$, Enjoji $M$, Ueo $H$ Sumachi $\mathrm{KI}$. Mesenteric panniculitis of the colon. Dis Colon Rectum 1987:30:962-6.

Simpson RD, Harrison EG, Mayo CW. Mesenteric fibromatosis in familial polyposis. Cancer 1964;17:526-34

8 Campbell JS, Ewing JB, Grice HC. Abdominal and pelvic paraffinomatosis: peritoneal and retro-peritoneal. Can $\mathcal{f}$ Surg 1958;1:131-41.

9 Soergel KH, Hensley GT. Fatal mesenteric panniculitis. Gastroenterology 1966;51:529-36.

10 Handelsman JC, Shelley WM. Mesenteric panniculitis. Arch Surg 1965;91:842-50.

\title{
Multinucleated stromal giant cells of the colonic lamina propria in ulcerative colitis
}

\author{
M A Pitt, W F Knox, N Y Haboubi
}

\author{
Department of \\ Histopathology, \\ Withington Hospital \\ Manchester M20 8LR \\ M A Pitt \\ W F Knox \\ N Y Haboubi \\ Correspondence to: \\ Dr M A Pitt \\ Accepted for publication \\ 22 April 1993
}

So-called (atypical) multinucleated stromal giant cells have been described in a variety of sites including the lower female genital tract, bladder, anus, skin, breast and nose. ${ }^{1-4}$ When numerous they have been confused with a diagnosis of sarcoma or pseudosarcomatous carcinoma. Their association with polyps, chronic inflammation and changes after radiation has suggested that these are reactive cells and studies suggest an origin from the indigenous stromal cells. ${ }^{1-4}$ An interaction between stromal cells and mast cells has been suggested as being crucial to the induction of this morphological change. ${ }^{1}$

\section{Case reports}

CASE 1

A 62 year old woman with an 18 year history of ulcerative proctitis that had been controlled by salazopyrine had a review colonoscopy at which the mucosa looked normal. Biopsy specimens were taken to assess disease activity and exclude dysplasia. 